\title{
Avoidance Task Training Potentiates Phasic Pontine-Wave Density in the Rat: A Mechanism for Sleep-Dependent Plasticity
}

\author{
Subimal Datta \\ Sleep Research Laboratory, Program in Behavioral Neuroscience, and Department of Psychiatry, Boston University School \\ of Medicine, Boston, Massachusetts 02118
}

\begin{abstract}
Behavioral studies of learning and memory in both humans and animals support a role for sleep in the consolidation and integration of memories. The present study explored possible physiological mechanisms of sleep-dependent behavioral plasticity by examining the relationship between learning and statedependent phasic signs of rapid eye movement (REM) sleep. Cortical electroencephalogram, electromyogram, eye movement, hippocampal $\theta$-wave, and pontine-wave ( $\mathrm{P}$-wave) measures were recorded simultaneously in freely moving rats after a session of conditioned avoidance learning or a control session. After learning trials, rats spent $25.5 \%$ more time in REM sleep and $180.6 \%$ more time in a transitional state between slow-wave sleep and REM sleep (tS-R) compared with that in control trials.
\end{abstract}

Both REM sleep and tS-R behavioral states are characterized by the presence of $\mathrm{P}$-waves. $\mathrm{P}$-wave density was significantly greater in the first four episodes of REM sleep after the learning session compared with the control session. Furthermore, the $\mathrm{P}$-wave density change between the first and third REM sleep episodes was proportional to the improvement of task performance between the initial training session and the postsleep retest session. These findings show that the increase in $\mathrm{P}$-wave density during the post-training REM sleep episodes is correlated with the effective consolidation and retention of avoidance task learning.

Key words: avoidance task training; brainstem; learning; plasticity; pontine-wave; pontogeniculooccipital wave; sleep
There is now considerable evidence of the involvement of sleep stages with memory processing and improvement of learning (for review, see Fishbein and Gutwein, 1977; McGrath and Cohen, 1978; Pearlman, 1979; Smith, 1985; Dujardin et al., 1990; Karni et al., 1994; Stickgold, 1998). To account for this sleep-dependent memory processing and learning, the following hypothesis has been suggested. During wakefulness, acquired information is stored temporarily in the hippocampus, amygdala, and some cortical and subcortical areas; during subsequent sleep, these areas are reactivated to "consolidate" acquired information into more permanent storage in the neocortex (Hennevin and Hars, 1985; Buzsaki, 1989, 1996; Pavlides and Winson, 1989; Wilson and McNaughton, 1994; Datta, 1999). These temporary storage areas would require a reactivating cue and/or triggering input during sleep (Bloch and Laroche, 1985; Bliss and Collingridge, 1993; Datta, 1999). The exact anatomical and physiological source of this cue and/or triggering input during natural sleep remains to be identified.

During rapid eye movement (REM) sleep and in part of slowwave sleep (SWS), phasic field potentials called pontogeniculooccipital (PGO) waves are generated in the pons (Brooks and Bizzi, 1963; Laurent and Ayalaguerrero, 1975; Sakai et al., 1976; Datta and Hobson, 1995). These phasic field potentials are a reflection of a phasic activation of a specific group of cells in the pons (Nelson et al., 1983; Datta et al., 1992, 1998; Datta and Hobson, 1994). PGO wave-generating cells fire as a high-frequency burst $(>500 \mathrm{~Hz})$ 25-30 msec before each PGO wave (Datta and Hobson, 1994). PGO waves have been recorded in discrete yet widespread regions along the neuraxis of rats, cats, and primates [for review, see Datta, 1997 (references)]. When these PGO waves are recorded from the pons of rats (Marks et al., 1980; Sanford et al., 1995) they are called

\footnotetext{
Received May 26, 2000; revised Aug. 24, 2000; accepted Aug. 30, 2000.

This work was supported by National Institutes of Health Grants NS 34004 and MH 59839. I gratefully acknowledge G. Buzsaki, J. Allan Hobson, E. H. Patterson, and R. Stickgold for their valuable discussions on this manuscript. I also thank Soma M. Datta for statistical analysis and for writing system software.

Correspondence should be addressed to Dr. Subimal Datta, Sleep Research Laboratory, Department of Psychiatry, Boston University School of Medicine, M-913, 715 Albany Street, Boston, MA 02118. E-mail: subimal@bu.edu.

Copyright (C) 2000 Society for Neuroscience $0270-6474 / 00 / 208607-07 \$ 15.00 / 0$
}

pontine waves (P-waves) (Datta et al., 1999). One recent anatomical study has demonstrated that functionally identified P-wave generator cells project to the hippocampus, amygdala, entorhinal cortex, visual cortex, and many other regions of the brain known to be involved in cognitive processing (Datta et al., 1998). Thus, the $\mathrm{P}$-wave-generating cells may serve as a trigger or cue for sleepdependent cognitive processes such as learning and memory (Datta, 1999). The present study demonstrates that the sleepdependent improvement of learning is correlated with the activation of a brainstem P-wave generation site.

\section{MATERIALS AND METHODS}

Subjects. Experiments were performed on 22 male Sprague Dawley rats (Charles River Laboratories, Wilmington, MA) weighing between 200 and $300 \mathrm{gm}$. The rats were housed individually at $24^{\circ} \mathrm{C}$ and given access to food and water ad libitum. Lights were on from 7:00 A.M. to 7:00 P.M. (light cycle) and off from 7:00 P.M. to 7:00 A.M. (dark cycle). Principles for the care and use of laboratory animals in research, as outlined by the National Institutes of Health (1985), were strictly followed.

Surgical procedures and implantation of electrodes. All surgical procedures were performed stereotaxically under aseptic conditions and were in accordance with the guidelines approved by the institutional animal care and use committee (IACUC protocol \#96-062). Animals were anesthetized (pentobarbital; $40 \mathrm{mg} / \mathrm{kg}$, i.p.), placed in a stereotaxic apparatus, and secured with blunt rodent ear bars. A surgical plane of anesthesia was maintained with supplemental injections of chloral hydrate $(60 \mathrm{mg} / \mathrm{kg}$, i.p.) every $1-2 \mathrm{hr}$, as necessary. The appropriate depth of anesthesia was judged by the absence of palpebral reflexes and by the absence of response to a tail pinch. Core body temperature was maintained at $37 \pm 1^{\circ} \mathrm{C}$ with a thermostatic heating pad and a rectal feedback thermister probe. The scalp was cleaned and painted with providone iodine (Betadine). A scalp incision was made, and the skin was retracted. The skull surface was cleaned in preparation for the implantation of electrodes. Potential postoperative pain was controlled with torbugesic (butorphanol tartrate; $0.5 \mathrm{mg} / \mathrm{kg}$, s.c.) To record the behavioral states of vigilance, electroencephalogram (EEG), electromyogram (EMG), and electrooculogram (EOG) electrodes were implanted. To record cortical EEG, stainless steel screw (jeweler's screw) electrodes connected to Teflon-coated stainless steel wires were screwed bilaterally into the skull $(2.0 \mathrm{~mm}$ anterior and $3.5 \mathrm{~mm}$ lateral to the bregma). An additional electrode was screwed into the skull $(4 \mathrm{~mm}$ anterior to the bregma in the midline) to act as a reference electrode. To record hippocampal EEG (for hippocampal $\theta$ waves), Teflon-coated bipolar stainless steel macroelectrodes were placed stereotaxically (Paxinos and Watson, 1986) in the hippocampus (anterior, 4.8; lateral, 2.5; H, 4.0 and 3.0). To record pontine EEG (for P-waves), ipsilateral to the hippocampal EEG-recording electrodes, Teflon-coated multipolar stainless steel macroelectrodes were placed stereotaxically in the pons (posterior, 
-0.80 ; lateral, $1.5 ; \mathrm{H}, 3.0,2.5,2.0$, and 1.5). A pair of Teflon-coated stainless steel wire electrodes was implanted bilaterally in neck muscles for recording nuchal EMG. To record eye movements (EOG), two Tefloncoated silver wire electrodes were implanted in the external canthus muscle of one eye. All electrodes were secured to the skull with dental acrylic. Electrodes were crimped to miniconnector pins and brought together in a plastic connector. Immediately after surgery, animals were placed in recovery cages and monitored for successful recovery from anesthesia and surgery. Successful recovery was gauged by the return of normal postures, voluntary movement, and grooming. At this point animals were transferred to their normal housing.

After a postsurgical recovery period of $3-7 \mathrm{~d}$, rats were habituated to a sound-attenuated recording cage (size, $76.2 \times 45.7 \times 45.7 \mathrm{~cm}$ ), shuttle box (size, $45.7 \times 20.3 \times 30.5 \mathrm{~cm}$; Shuttle Scan; model SCII; AccuScan Instruments, Columbus, $\mathrm{OH}$ ), and free-moving polygraphic recording conditions for $7 \mathrm{~d}$.

Avoidance learning. The apparatus is an automated two-way shuttle scan shock-avoidance box (size, $45.7 \times 20.3 \times 30.5 \mathrm{~cm}$ ) with sides made of high-grade acrylic. The floor is made of stainless steel bars suitable for application of shock. The box is bisected by a vertical partition with an opening in the middle (near the bottom). This opening permits the animal to travel freely from one side of the shuttle box to the other. The box contains a front and a rear sensor containing eight infrared light beams. These light beams determine positively which side the animal is on. Located on the lid of the shuttle box are three light bulbs (one in each compartment and one in the center) that provide light stimuli (adjustable intensity, $6 \mathrm{~W}$ at $115 \mathrm{~V} \mathrm{AC}$ ) and three beepers (3600 Hz; adjustable $0.00-85 \mathrm{~dB}$ at $30 \mathrm{~cm}$ ) that produce sound stimuli. The interface unit permits interconnections between the computer and the shuttle box. A personal computer using remote monitoring system software controls experimental protocols and data collections. By the use of the Omnitech Shuttle Box Monitoring System, the computer controls the shuttle box for both conditioned and unconditioned stimulus parameters. The same software and computer also collect and analyze avoidance data on-line.

The procedure involved placing the rat in one compartment of the apparatus. After $15 \mathrm{~min}$ of acclimatization, training trials were begun. During acclimatization and the training trials, the rats could move freely from one compartment to the other within the shuttle box. Rats were trained on a massed 30-trial shuttle box two-way active avoidance task. The procedures for the conditioned stimulus (CS) and unconditioned stimulus (UCS) paired group (CS-UCS paired learning group) were as follows. A tone $(3600 \mathrm{~Hz} ; 45 \mathrm{~dB})$ and a pulsatile light $(2.5 \mathrm{~Hz})$ were presented as a CS in the compartment with the animal, paired $5 \mathrm{sec}$ later with a $0.3 \mathrm{~mA}$ scrambled foot shock (UCS) delivered through the floor grid (steel rods 0.5 $\mathrm{cm}$ in diameter, spaced $1.5 \mathrm{~cm}$ between centers). To avoid receiving a foot shock the rat had $5 \mathrm{sec}$ to move to the opposite compartment. If the animal did not move to the other compartment, UCS was delivered for a maximum of $5 \mathrm{sec}$, and CS ended with UCS. While receiving UCS, if the animal moved to the other compartment, both CS and UCS ended immediately. The intertrial interval was variable with a mean of $60 \mathrm{sec}$. A group of CS-UCS random control rats received 30 trials of CS $(10 \mathrm{sec}$ in duration) and 10 trials of randomly delivered UCS ( $5 \mathrm{sec}$ in duration) that they could not avoid even by moving to the other compartment of the shuttle box.

Since the discovery of REM sleep, animal studies of sleep and learning have used various hippocampally and/or nonhippocampally mediated learning paradigms (for review, see Smith, 1985; Stickgold, 1998). In this study, we have used a two-way active avoidance learning task that involves both hippocampal and nonhippocampal structures for learning and memory processing (Smith and Young, 1980; Ambrosini et al., 1988; Ramirez and Carrer, 1989; Bramham et al., 1994). The involvement of the hippocampus and some nonhippocampal structures in learning and memory processing is supported by many other studies (Squire et al., 1990; LeDoux, 1992; Silva et al., 1992; Izquierdo et al., 1995; Hatfield et al., 1996; Rempel-Clower et al., 1996; Poremba and Gabriel, 1997; Young et al., 1997; Gallagher et al., 1999; Vazdarjanova and McGaugh, 1999). One recent anatomical study provided evidence that P-wave-generating cells project monosynaptically to both the hippocampus and nonhippocampal structures involved in the learning process (Datta et al., 1998). Because P-wave-generating cells project to both hippocampal and nonhippocampal structures, the activation of P-wave-generating cells may modulate both hippocampally and nonhippocampally mediated learning processes. Therefore, the selection of a two-way active avoidance learning task, which involves both hippocampal and nonhippocampal structures, was appropriate to study the relationship between P-waves and learning. In the past, many other sleep and learning studies used both one-way and two-way active avoidance learning tasks (Joy and Prinz, 1969; Albert et al., 1970; Wolfowitz and Holdstock, 1971; Smith and Butler, 1982; Ambrosini et al., 1988; Smith and Kelly, 1988; Ramirez and Carrer, 1989; Bramham et al., 1994). All of these active avoidance studies demonstrated a crucial increase in REM sleep when learning occurred. This increase in REM sleep is critical for the improvement of learning in the retest session. In the present experimental protocol the control and learning groups of animals were exposed to identical training sessions except that the CS and UCS were unpaired for the control group, making avoidance learning impossible.

Determination of behavioral states. For the purpose of determining possible effects on sleep and wakefulness, four behavioral states were distin- guished on the basis of polygraphic data. These four states were as follows: (1) Wakefulness (W): low-voltage $(50-80 \mu \mathrm{V})$ fast $(30-50 \mathrm{~Hz})$ cortical EEG, high-amplitude tonic and phasic EMG bursts, presence of eye movements in the EOG, gross bodily movements, and an absence of P-waves. (2) SWS: spindling and high-voltage $(200-400 \mu \mathrm{V})$ slow waves $(0.3-15 \mathrm{~Hz})$ in the cortical EEG, EMG tonus lower than during W, absence of eye movements, and absence of P-waves. (3) Transition state between SWS and REM sleep (tS-R): stage of sleep appearing between SWS and REM sleep. The tS-R sleep stage always precedes REM sleep onset but itself is not always followed by REM sleep. During this stage, cortical EEG is a mixture of partly low-amplitude $(50-80 \mu \mathrm{V})$, high-frequency $(15-25$ $\mathrm{Hz})$ and high-amplitude $(200-300 \mu \mathrm{V})$, low-frequency $(5-10 \mathrm{~Hz})$ waves. The EMG tone is absent or progressively diminished. Eye movements are absent in the EOG record. $\theta$ Frequency waves start to appear in the hippocampal EEG. Spiky P-waves (10-20 per min) start to appear in the pontine EEG. These P-waves are mostly the single-spike type. (4) REM sleep: low-voltage $(50-100 \mu \mathrm{V})$ and fast $(20-40 \mathrm{~Hz})$ cortical EEG, presence of muscle atonia, rapid eye movements, and $\theta$ waves $(4-7 \mathrm{~Hz})$ only in the hippocampal EEG, and increased occurrence of P-waves, most of them occurring in clusters of two to three. States were scored in $10 \mathrm{sec}$ epochs. Simultaneous recordings of cortical EEG, hippocampal $\theta$ wave, EMG, EOG, and P-waves ensured accurate identification of behavioral states in this study. Because the activation of specific brainstem nuclei generates discrete tonic and phasic signs of REM sleep (Vertes, 1984; Datta, 1995), simultaneous recording of these signs enabled the identification of changes in the level of activation of specific brainstem structures in relation to the learning trials.

Experimental protocol. After the adaptation sessions, rats underwent sessions of baseline sleep-wakefulness recordings on 3 consecutive days. During baseline recordings, rats were placed in the shuttle box for $45 \mathrm{~min}$ (9:10-9:55 A.M.) and then transferred to a recording cage for $6 \mathrm{hr}$ of polygraphic recordings (between 10:00 A.M. and 4:00 P.M.). On the day after the final of the three baseline recording sessions, rats were randomly assigned to either the CS-UCS paired paradigm (learning test; $n=12$ rats) or the CS-UCS unpaired paradigm (control for the learning test; $n=$ 10 rats). Rats were placed in the shuttle box at 9:10 A.M., and after $15 \mathrm{~min}$ of an acclimatization period, CS trials began. After 30 trials, rats were transferred to the polygraphic recording cage and recorded for $6 \mathrm{hr}$ (between 10:00 A.M. and 4:00 P.M.) sessions of undisturbed sleep-wakefulness. At the end of the $6 \mathrm{hr}$ recordings, the learning group was retested for 30 CS-UCS learning trials (between 4:05 and 4:50 P.M.).

Data analysis. The polygraphic measures provided the following dependent variables that are quantified for each trial: (1) percentage of recording time spent in W, SWS, tS-R, and REM sleep, (2) latencies to onset of the first episodes of tS-R and REM sleep after the onset of recordings, (3) total number of $t S-R$ and REM sleep episodes, (4) mean duration of tS-R and REM sleep episodes, (5) P-wave density (waves/minute) in REM sleep, (6) $\theta$-wave frequency (waves/second) in REM sleep, and (7) REM density (waves/minute) in REM sleep. For latency analysis, the data collection began immediately after the rats were transferred to the recording cage and connected to the recording cable. All of these variables during baseline recording days (before shuttle box trials) were analyzed by the use of two-way ANOVAs (group $\times$ day), with day as a repeated measure, using StatView statistical software (Abacus Concepts, Berkeley, CA). These analyses were performed to be sure that the learning and control groups were not different before the learning trials and that there were no variations between days. After shuttle box trials, all of the above variables were analyzed for the $6 \mathrm{hr}$ recording sessions by the use of one-factor ANOVAs (learning vs control group) to determine the effect of avoidance learning.

For the analysis of performance on the two-way active avoidance learning task, the 30 learning trials were divided into six blocks of five trials, and the percentage of successful avoidances was calculated for each block. These data were then analyzed by the use of two-way ANOVA (session $x$ block), with block as a repeated measure, and followed by a Scheffe post hoc $F$ test. The level of significance was set at $p<0.05$. These analyses were performed to determine the differences in learning curves between the first session (morning trials, before the $6 \mathrm{hr}$ undisturbed polygraphic recordings) and the second session (after $6 \mathrm{hr}$ polygraphic recordings). This difference represents a quantitative measure of the amount of information retained from the first learning session. The improvement of performance between training trials (first session) and retest trials (second session) was calculated by subtracting the percentage of avoidance in the first two blocks of training from the percentage of avoidance in the first two blocks of retest.

\section{RESULTS}

\section{Effect of avoidance learning on wake-sleep states}

In the group of learning animals, statistical comparisons (two-way ANOVAs) between the 3 baseline recording days revealed no significant differences in the total percentage of time spent in W, SWS, tS-R, and REM sleep between the $6 \mathrm{hr}$ periods. Similarly, the percentage of time the control group spent in W, SWS, tS-R, and REM sleep during the $3 \mathrm{~d}$ of baseline recording did not significantly 


\begin{tabular}{|c|c|c|}
\hline Variable & $\begin{array}{l}\text { CS-UCS unpaired } \\
\text { (Control group) }\end{array}$ & $\begin{array}{l}\text { CS-UCS paired } \\
\text { (Learning group) }\end{array}$ \\
\hline Wakefulness (\%) & $35.0 \pm 4.4$ & $33.9 \pm 4.7$ \\
\hline Slow-wave sleep (\%) & $52.2 \pm 5.8$ & $47.3 \pm 5.5$ \\
\hline Transition (tS-R) (\%) & $1.4 \pm 0.5$ & $3.9 \pm 0.5^{* * *}$ \\
\hline Latency (min) & $28.5 \pm 7.3$ & $23.4 \pm 6.5$ \\
\hline Total number (in 6 & & \\
\hline hr) & $28.8 \pm 9.7$ & $64.6 \pm 8.2^{* * *}$ \\
\hline Duration (sec) & $10.8 \pm 7.9$ & $12.3 \pm 5.9$ \\
\hline REM sleep (\%) & $11.8 \pm 2.3$ & $14.8 \pm 2.4^{* *}$ \\
\hline Latency (min) & $41.6 \pm 7.3$ & $37.4 \pm 6.5$ \\
\hline Total number (in 6 & & \\
\hline hr) & $15.8 \pm 1.5$ & $21.6 \pm 1.9^{* * *}$ \\
\hline Duration (sec) & $147.8 \pm 47.9$ & $152.3 \pm 55.9$ \\
\hline \multicolumn{3}{|l|}{$\begin{array}{l}\text { P-wave density in REM } \\
\text { sleep (waves } / \mathrm{min} \text { ) }\end{array}$} \\
\hline Episode 1 & $39.2 \pm 3.4$ & $53.0 \pm 6.4^{* * *}$ \\
\hline Episode 2 & $38.0 \pm 3.0$ & $60.2 \pm 7.3^{* * *}$ \\
\hline Episode 3 & $39.6 \pm 4.2$ & $64.0 \pm 8.4^{* * *}$ \\
\hline Episode 4 & $40.4 \pm 2.8$ & $53.0 \pm 5.5^{* * *}$ \\
\hline Episode 5 & $40.4 \pm 3.8$ & $43.3 \pm 4.4$ \\
\hline Episode 6 & $39.0 \pm 3.8$ & $40.5 \pm 3.7$ \\
\hline $\begin{array}{l}\text { REM density in REM } \\
\text { sleep (waves/min) }\end{array}$ & $47.6 \pm 10.3$ & $52.4 \pm 9.5$ \\
\hline $\begin{array}{l}\theta \text {-Wave frequency in } \\
\text { REM sleep }(\mathrm{Hz})\end{array}$ & $6.33 \pm 0.6$ & $6.30 \pm 0.5$ \\
\hline
\end{tabular}

The wakefulness, slow-wave sleep, transition, and REM sleep values were analyzed from the $6 \mathrm{hr}$ period of undisturbed sleep recorded immediately after control (CSUCS unpaired) or learning (CS-UCS paired) trials. The P-wave density, REM density, and $\theta$-wave frequency were analyzed from the first six episodes of REM sleep after control or learning trials.

Post hoc $t$ tests: **p $<0.01 ;{ }^{* * *} p<0.001$.

change. Because there was no day effect, the last baseline day was used for the comparison between control and learning groups. Post hoc tests (one-factor ANOVAs) found no significant difference between control and learning groups before learning trials for the total percentage of W $(35.1 \pm 3.3$ vs $34.8 \pm 2.9 \%)$, SWS $(50.8 \pm 3.9$ vs $51.5 \pm 2.6 \%)$, tS-R $(1.8 \pm 0.4$ vs $1.8 \pm 0.5 \%)$, or REM sleep $(12.3 \pm 1.2$ vs $11.9 \pm 1.1 \%)$. In addition, P-wave density $(38.7 \pm 4.1$ vs $40.5 \pm 3.8)$, REM density ( $44.6 \pm 9.8$ vs $47.3 \pm 10.6)$, and $\theta$-wave frequency $(6.26 \pm 0.7$ vs $6.29 \pm 0.8)$ during REM sleep were not significantly different (one-factor ANOVA) between groups. Thus, the groups were initially equal in terms of time spent in W, SWS, tS-R, and REM sleep, P-wave density, $\theta$-wave frequency, and REM density for the final $6 \mathrm{hr}$ baseline recording period.

The rats did not show any overt abnormal waking behavior after the shuttle box trials. The percentages of wakefulness after shuttle box trials (control, $35.0 \pm 4.4 \%$; learning, $33.9 \pm 4.7 \%$ ) were similar and comparable with the percentages before the trials. After the trials, the total percentage of wakefulness in the learning group was not significantly different when compared with that of the control group $\left[F_{(1,20)}=0.275 ; p=0.6059\right.$; Table 1]. After the shuttle box trials the mean percentage of time spent in SWS in the learning group $(47.3 \pm 5.5 \%)$ was slightly less than that of the control group $(52.2 \pm 5.8 \%)$, but these differences were not statistically significant $\left[F_{(1,20)}=4.147 ; p=0.0552\right.$; Table 1; Fig. 1].

The behavioral state of $\mathrm{tS}-\mathrm{R}$ is a very short stage of sleep that appears between SWS and REM sleep and lasts for $\sim 5-20$ sec. The tS-R always precedes REM sleep onset but is not always followed by REM sleep. The total percentage of $t S-R$ in the post-trial

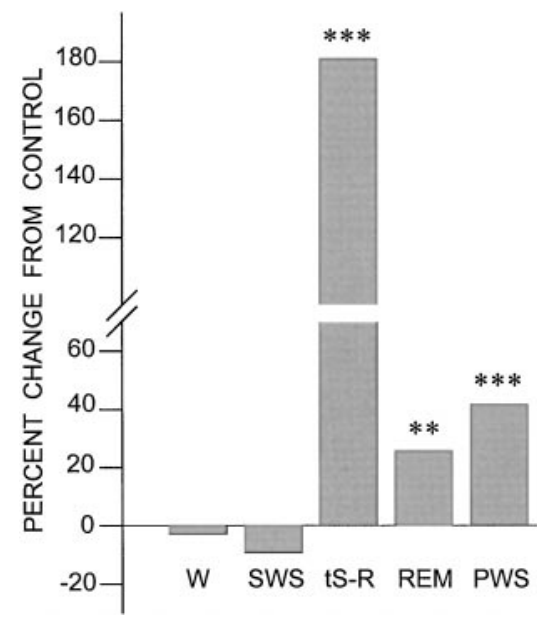

Figure 1. Effects of learning trials compared with control trials on wakefulness and different sleep states. Percentage changes in wakefulness $(W)$, slow-wave sleep $(S W S)$, transition from slow-wave sleep to REM sleep $(t S-R)$, REM sleep $(R E M)$, and total P-wave state $(P W S$; combined $t S-R$ and $R E M)$ from the first session of control trials ( $O$ line $)$ to the first session of learning trials (CS-UCS paired). Note that the percentage of change from the control trials (CS-UCS unpaired) in $W$ and $S W S$ after the learning trials (CS-UCS paired) is not significant. However, the learning trials significantly increased the percentage of $t S-R, R E M$, and $P W S$ from the control trials. Post hoc $t$ tests: ${ }^{* *} p<0.01 ;{ }^{* * *} p<0.001$.

controls $(1.4 \pm 0.5 \%)$ was comparable with the total percentage of pretrial controls $(1.8 \pm 0.4 \%)$. After the shuttle box trials, the total percentage of time spent in $\mathrm{tS}-\mathrm{R}$ in the learning group of rats was significantly higher [180.57\% higher; $\left.F_{(1,20)}=136.123 ; p<0.001\right]$ compared with the control rats (Table 1; Fig. 1). Having documented the post-trial increase in the total percentage of $t S-R$ in the learning group, we looked at the latency, frequency, and duration of tS-R episodes (Table 1). The mean latency of tS-R in the learning group $(23.4 \pm 6.5 \mathrm{~min})$ was not significantly different from that of the control group $(28.5 \pm 7.3 \mathrm{~min})$. Similarly, the mean duration of tS-R $(10.8 \pm 7.9 \mathrm{sec})$ was not significantly different between groups. But the mean number of tS-R episodes in the learning group was significantly higher $\left[124 \%\right.$ higher; $F_{(1,20)}=$ 142.324; $p<0.001]$ than that in the control group.

Figure 2 illustrates the polygraphic characteristics of typical post-trial REM sleep episodes of the control (Fig. $2 A$ ) and learning (Fig. $2 B$ ) groups. After the shuttle box trials, the total percentage of time spent in REM sleep in the learning group was significantly higher [25.4\% higher; $\left.F_{(1,20)}=9.053 ; p<0.01\right]$ than that in the control group (Table 1; Fig. 1). Having documented a significant increase in post-trial REM sleep in the learning group, we looked at latency, duration, and frequency of REM sleep episodes (Table 1). The mean latency of REM sleep in the learning group (37.4 \pm $6.5 \mathrm{~min}$ ) was not significantly different from that of the controls $(41.6 \pm 7.3 \mathrm{~min})$. Similarly, the mean duration of REM sleep was not significantly different. But, again, the mean number of REM sleep episodes in the learning group of animals was significantly higher [36.7\% higher; $\left.F_{(1,20)}=105.243 ; p<0.001\right]$ than that in the controls (Table 1; Fig. 1).

\section{Effect of avoidance learning on the P-wave state and P-wave density}

Because P-waves are normally present only during the behavioral states of tS-R and REM sleep, we have referred to these as the P-wave state (PWS) (Datta et al., 1992). The combined mean percentage of PWS after learning trials in the learning group $(18.7 \pm 2.3 \%)$ was significantly higher $\left[41.7 \%\right.$ higher; $F_{(1,20)}=$ $26.635 ; p<0.001]$ than that in the control $(13.2 \pm 2.7 \%)$ after control trials (Fig. 1). Having documented a significant increase in the post-trial PWS percentage in the learning group compared with the control, we looked at P-wave density during the first six REM 


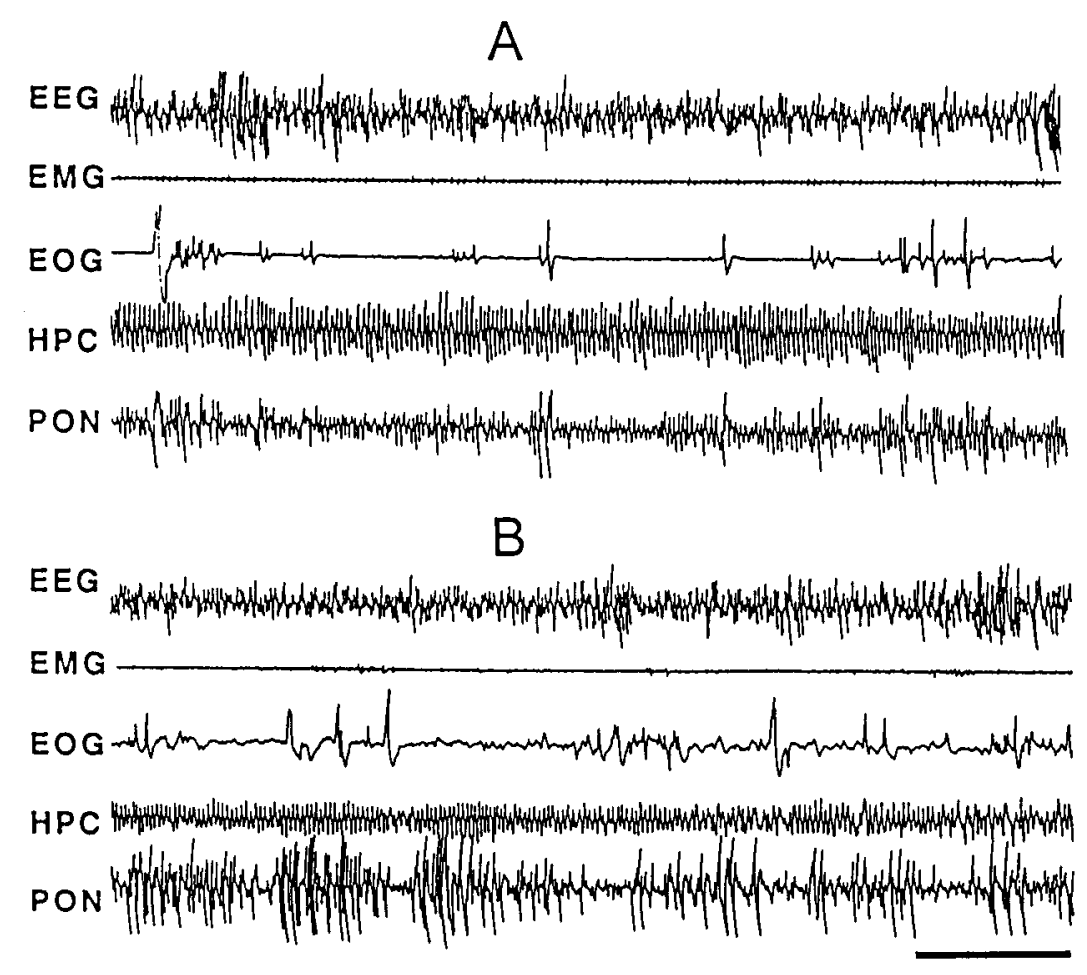

Figure 2. Sample polygraphic appearance of the third episode of REM sleep after the first session of control trials $(A)$ and learning trials $(B)$. Note the qualitative similarity in both records showing characteristic electrographic signs of REM sleep: low-voltage, high-frequency, or desynchronized waves recorded from the frontal cortex $(E E G)$; muscle atonia $(E M G)$; rapid eye movements $(E O G)$; hippocampal $\theta$ waves in the hippocampal EEG (HPC); and P-waves (spiky waves) in the pontine $E E G(P O N)$. Despite qualitative similarity, $\mathrm{P}$-waves are more frequent in the learning trials than in the control trials. Time scale, $5 \mathrm{sec}$.

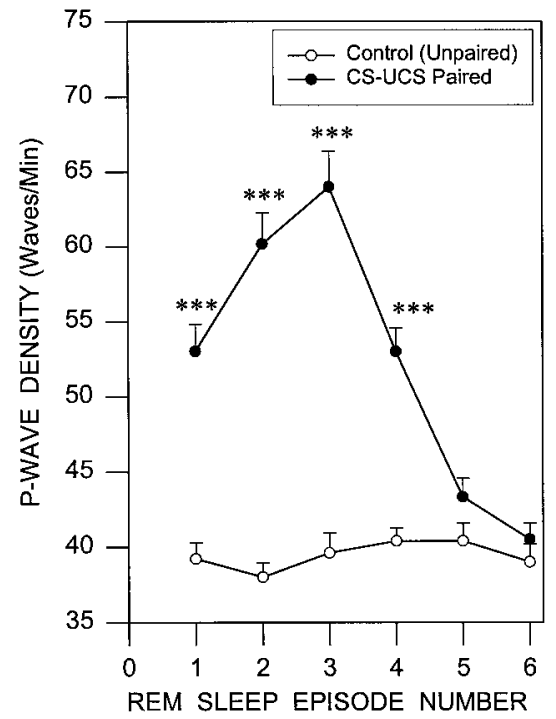

Figure 3. Increased P-wave density in the first four episodes of REM sleep after a session of learning trials. This line graph illustrates P-wave density (mean $\pm \mathrm{SE}$ ) in the first six episodes of REM sleep after a session of the control trials (empty circles) and learning trials ( filled circles). Note that the $\mathrm{P}$-wave density in the first four episodes of REM sleep is significantly higher after the learning trials compared with the control trials. Also note that the P-wave density sharply increased from the first episode of REM sleep to the third episode in which it peaked and then started to decline toward the baseline density level. Post hoc $t$ tests: ${ }^{* *} p<0.001$.

sleep episodes after the shuttle box avoidance learning and control trials (Fig. 3). Statistical analysis (two-way ANOVA) showed a significant difference in P-wave density between groups $\left[F_{(1,20)}=\right.$ 50.16; $p<0.001]$ and between REM sleep episodes $\left[F_{(5,100)}=\right.$ 52.95; $p<0.001]$. Post hoc analysis (one-factor ANOVA) identified that the P-wave densities in the learning animals were significantly higher than those of the control group during REM sleep episodes $1\left[F_{(1,20)}=37.801 ; p<0.001\right], 2\left[F_{(1,20)}=80.284 ; p<0.001\right], 3$ $\left[F_{(1,20)}=70.108 ; p<0.001\right]$, and $4\left[F_{(1,20)}=43.04 ; p<0.001\right]$ but not during episodes 5 and 6 (Table 1 ).

\section{Effect of avoidance learning on the $\theta$-wave frequency and REM density}

In the cat, REMs are highly correlated with PGO activity (Nelson et al., 1983; Datta and Hobson, 1994). Having documented robust change in P-wave density after learning trials, we have looked at REM density during the first six REM sleep episodes after learning and control trials. The mean REM density in the learning group (52.4 \pm 9.5 waves $/ \mathrm{min})$ when compared with that of the control group (47.6 \pm 10.3 waves/min) was not significantly different. We also compared $\theta$-wave frequency in the first post-trial REM sleep episodes. Again $\theta$-wave frequency in the learning group (6.30 \pm $0.53 \mathrm{~Hz}$ ) was not significantly different from that of the control group $(6.33 \pm 0.63 \mathrm{~Hz})$.

\section{Improvements in learning}

Performance on the shuttle box avoidance task is shown in Figure 4. The learning group was subjected to two sessions of learning trials-one before and another after the $6 \mathrm{hr}$ session of undisturbed polygraphic sleep recordings. For each of the two sessions, the percentage of conditioned avoidance responses was calculated for each rat for each of the six blocks of five trials and than averaged across animals. In the training session, rats performed poorly in the first two blocks of trials. In the third block of trials, animals avoided approximately one-third $(38.33 \%)$ of the UCS. By the fifth and sixth blocks of trials these animals successfully avoided $>80 \%$ of the UCS. In contrast, at retest rats avoided $>50 \%$ of the UCS during the first two blocks, and by the third block animals avoid $>80 \%$ of UCS. Two-way ANOVA (session $\times$ block) revealed a rapid and significant increase in the percentage of avoidance responses over blocks $\left[F_{(5,110)}=84.38 ; p<0.001\right]$ as they acquired the task. Overall the percentage of avoidance was significantly greater in session 2 than in session $1\left[F_{(1,22)}=61.25 ; p<0.001\right]$. Post hoc analysis (one-factor ANOVA and Scheffe $F$ test) revealed a significantly higher percentage of avoidance in the second session for each of the first four blocks (Fig. 4).

\section{Relationship between P-wave density and improvement in avoidance learning}

Because there was a sharp rise of P-wave density between the first and third episodes of REM sleep after the training session (Fig. 3), we expected to see a precise relationship between the increase in 


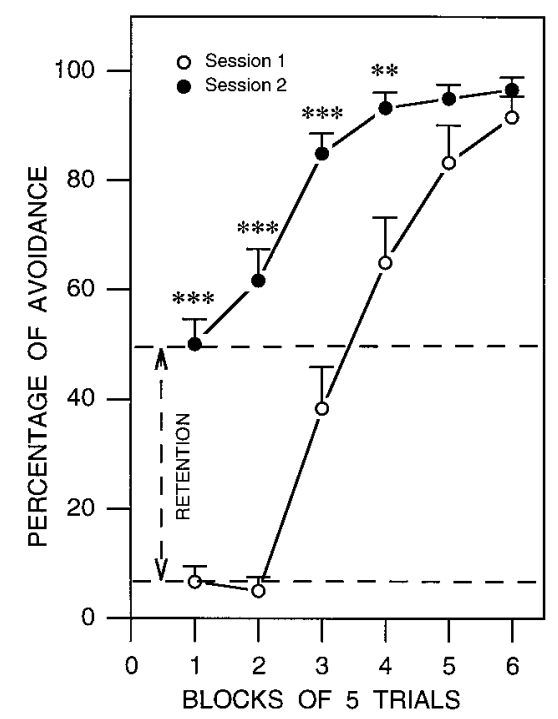

Figure 4. Learning curves in session 1 (empty circles) and session 2 ( filled circles) for the two-way active avoidance task. The percentage of avoidance learning (mean $\pm \mathrm{SE}$ ) is plotted here in blocks of five trials. Note that in the first session the rats $(n=12)$ performed poorly in the first two blocks of trials (10 trials) and then in the third block the animals started to avoid. By the sixth block the animals successfully avoided in $>80 \%$ of the trials. After the first session the animals were allowed $6 \mathrm{hr}$ of undisturbed sleep. After sleep, the animals were subjected to the second session of active avoidance trials. Unlike in the first session, during the first two blocks of the second session, animals avoided in $>50 \%$ of the trials. These data indicate the improvement in the avoidance learning (or retention) between session 1 and session 2. By the third block of trials animals avoided in $>80 \%$ of the trials. Post hoc $t$ test: ${ }^{* *} p<0.01 ; * * p<0.001$.

P-wave density between the first and third REM sleep episodes and the improvement in performance. Indeed, a strong correlation was observed (Pearson correlation coefficient, $r=0.95$; $\mathrm{df}=11 ; p<$ 0.001; Fig. 5). Similarly, the P-wave density change between the last baseline recording session and the first post-training REM sleep period also showed a statistically significant positive slope $(r=$ $0.70 ; \mathrm{df}=11 ; p<0.01)$. These results suggest that the increase in $\mathrm{P}$-wave density in the third REM sleep episode is correlated with effective task performance.

\section{DISCUSSION}

The principal findings of this study are that (1) avoidance task training increased the time spent in the P-wave-related behavioral states of tS-R and REM sleep during the $6 \mathrm{hr}$ after training but did not alter the time spent in W and SWS, (2) training increased P-wave density in the first four episodes of REM sleep in the post-training period but had no effect on hippocampal $\theta$ frequency or rapid eye movement density during post-training REM sleep, and (3) improved performance in the retrial session was proportional to the increase in P-wave density during the first three REM sleep episodes after task training. These results suggest a strong relationship between $\mathrm{P}$-waves and state-dependent information processing that may have relevance for our understanding of the physiological mechanisms underlying learning and memory during sleep.

The present study demonstrates that during the post-trial sleeprecording session rats spent $25.47 \%$ more time in REM sleep after learning trials than after control trials. This increase in total REM sleep time was caused by an increase in the number of REM sleep episodes during the post-training period. These results agree with previous animal studies, using a variety of protocols and test paradigms, that have consistently shown that both appetitive and aversive training increases REM sleep in the post-training period (Lucero, 1970; Fishbein et al., 1974; Smith et al., 1980; PortellCortes et al., 1989; Smith and Wong, 1991; Bramham et al., 1994; Smith and Rose, 1997). These increases in REM sleep appear not to be caused simply by the stress of the training protocol but to be

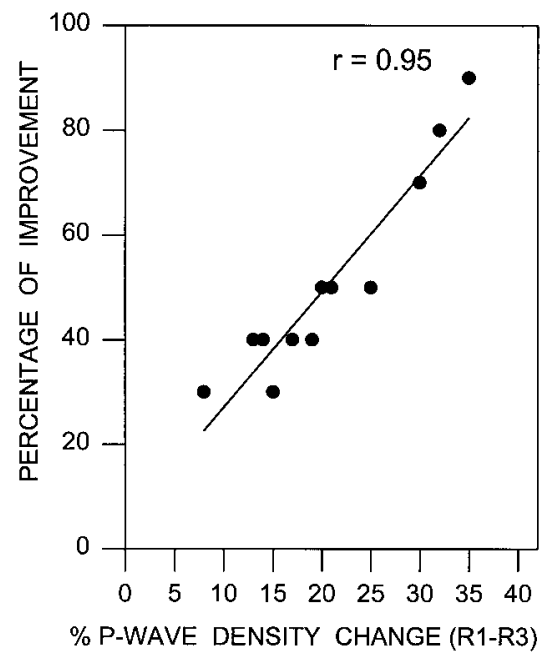

Figure 5. Relationship between the P-wave density change and the improvement in learning. The percentage of improvement for each animal (filled circles) is shown as a function of the percentage of P-wave density change between the first $(R 1)$ and third $(R 3)$ episodes of REM sleep after the first session of active avoidance learning trials $(n=12$ rats). The percentage of improvement was calculated by subtracting the percentage of avoidance in the first two blocks of the first session of learning trials from the percentage of the first two blocks in the second session. The plot of linear regression best-fit (solid line; Pearson product-moment correlation) shows a statistically significant positive slope $(r=0.95 ; p<0.001)$. These data indicate that the level of improvement of learning in the retrial session depends positively on the percentage of the P-wave density increase in between the first and third episodes of REM sleep immediately after the first session of learning trials.

caused by active learning of new material (Hennevin et al., 1995). Because of the absence of a REM sleep change in those animals that received foot shocks noncontingently (control group), the increase in REM sleep in the animals that received learning trials cannot simply be attributed to the stress of foot shocks. Other animal studies have also demonstrated that post-training REM sleep deprivation can partially or even totally block improved task performance on subsequent retesting (Fishbein, 1971; Pearlman, 1973; Pearlman and Becker, 1973; Smith and Butler, 1982; Smith et al., 1998). Taken together, these animal studies suggest that the increased REM sleep after acquisition is critical for learning. In the current study we also observed that the learning group spent $180.57 \%$ more time in tS-R (SWS with P-waves) than did the control group during the post-trial sleep-recording session. Again, this increase in tS-R was caused mainly by an increase in the total number of $\mathrm{tS}-\mathrm{R}$ events. After spatial exploration, activity patterns of hippocampal place cells have been shown to be reactivated during subsequent SWS, and this replay is believed to contribute to memory formation and consolidation (Pavlides and Winson, 1989; Wilson and McNaughton, 1994; Kudrimoti et al., 1999). Because these studies did not record P-wave activity, it is possible that the hippocampal reactivation episodes were in fact during $\mathrm{tS}-\mathrm{R}$. So, the increase in $\mathrm{tS}-\mathrm{R}$ may be caused by a increase in demand for the cellular reactivation and memory processing.

Our finding that two-way active avoidance learning trials do not change significantly the total amount of SWS agrees with previous sleep learning studies using other types of learning paradigms that have similarly shown that learning trials do not have an effect on the amount of post-trial SWS (Lucero, 1970; Fishbein et al., 1974; Smith and Rose, 1997). These findings suggest that there is no demand for increased SWS for post-trial memory consolidation. Interpretation of these behavioral studies is also supported by several lines of evidence from physiological studies (Bloch and Laroche, 1981, 1984; Hars et al., 1985; Hars and Hennevin, 1987; Jones-Leonard et al., 1987; Bramham and Srebro, 1989; Maho and Bloch, 1992).

On the basis of a number of neurophysiological studies, off-line reactivation of various neuronal structures involved in learning 
seems to be critical for the consolidation of memories (Pavlides and Winson, 1989; Wilson and McNaughton, 1994; Skaggs and McNaughton, 1996; Qin et al., 1997; Kudrimoti et al., 1999). This reactivation hypothesis of memory consolidation is also supported by a number of electrical stimulation studies (Stein and Chorover, 1968; Erickson and Patel, 1969; Destrade et al., 1973; Landfield et al., 1973; Destrade and Cardo, 1974). These studies have shown that mice and rats receiving post-trial hippocampal stimulation showed better retention of learning than did control animals. These studies also showed that when the hippocampus was reactivated by electrical stimulation there was no need for sleep for the improvement of learning. In summary, reactivation of the hippocampus is critical for sleep-dependent memory processing. In the present study sleep-dependent memory processing may involve reactivation of other brain regions in addition to the hippocampus. To differentiate between the role of reactivation of other forebrain structures and the hippocampus, future studies will use tasks that differentially involve the hippocampus and other brain regions, especially tasks that use the amygdala.

If reactivation of forebrain and cortical memory-processing networks is critical for the consolidation of memory, then what is the source of this reactivating stimulus during tS-R and REM sleep? This reactivating stimulus is probably coming from the brainstem. This possibility is supported by the finding that the electrical stimulation of the mesencephalic reticular formation (MRF) after training improves performance in the rat (Leconte et al., 1974; DeWeer, 1976; Bloch et al., 1977; Devietti et al., 1977; Sara et al., 1980; Bloch and Laroche, 1981; Hennevin et al., 1989). The improvement in learning performance by post-trial MRF stimulation was as effective as hippocampal stimulation. A post-trial MRF stimulation was shown to facilitate a classically conditioned association and also the development of associative changes in neuronal activity in the hippocampus as the conditioning proceeded (Bloch and Laroche, 1981, 1984). Moreover, when stimulation was administered after each long-term potentiation (LTP)-inducing stimulus, it enhanced the magnitude of LTP at the synapses of the perforant path on dentate granular cells and prolonged its duration by several days. The MRF stimulation during the postacquisition period appeared to substitute the need for REM sleep by decreasing the post-training REM sleep elevation and by abolishing most of the learning impairment produced by post-trial REM sleep deprivation (Bloch et al., 1977). This leads to the assumption that MRF stimulation during REM sleep has enhanced an ongoing physiological process that naturally occurs during postlearning REM sleep and that could be responsible, at least partially, for the beneficial effect of postlearning REM sleep on memorization. On the basis of the above evidence, it may be proposed that during REM sleep the MRF is the source of the reactivating stimulus for the memory-processing network in the forebrain and cortical areas. However, these MRF electrical stimulation studies did not systematically localize a most effective or only effective site in the MRF for the reactivation of the memory-processing network. Is the whole MRF or specific nuclei the source of reactivation? It is likely that electrical stimulation of the MRF in these studies also stimulated other parts of the brainstem reticular formation.

The MRF is an important part of the brainstem reticular formation, and it contains a number of specific cell groups involved in the generation of different signs of REM sleep (for review, see Datta, 1995). During REM sleep, different parts of the brainstem reticular formation are activated for the generation of different phasic and tonic signs of REM sleep (for review, see Vertes, 1984; Datta, 1995, 1997). Which cell group(s) or structure(s) in the brainstem is responsible for the reactivation of the memory-processing network in the forebrain and cortex? The present study demonstrated that there was an increase in tS-R and REM sleep after learning trials. Because $\mathrm{P}$-wave-generating cells are activated only during $\mathrm{tS}-\mathrm{R}$ and REM sleep and P-wave-generating cells have anatomical projections to the forebrain and cortical memory-processing structures (Datta et al., 1998), the activation of P-wave-generating cells could reactivate the memory-processing network. The present study also demonstrated that learning trials increase P-wave density in the subsequent four REM sleep episodes. In addition, this study has shown that the improvement of learning in a retrial session is proportional to the rate of $\mathrm{P}$-wave density change between the first and third REM sleep episodes after the first session of learning trials. These findings suggest that a major source of this reactivating stimulus is the phasic P-wave-generating cells.

In conclusion, activation of $\mathrm{P}$-wave-generating cells during $\mathrm{tS}-\mathrm{R}$ and REM sleep may reactivate the forebrain and cortical memoryprocessing structures to reprocess recently stored information, aiding in the maintenance of memory and facilitating its later expression. Depending on the demand of learning, supplementary activation of P-wave-generating cells could further enhance the information-processing efficiency, resulting in improved performance.

\section{REFERENCES}

Albert IB, Cicala GA, Siegal J (1970) The behavioral effects of REM sleep deprivation in rats. Psychophysiology 6:550-560.

Ambrosini MV, Sadile AG, Gironi Carnevale UA, Mattiaccio A, Giuditta A (1988) The sequential hypothesis on sleep function. II. A correlative study between sleep variables and newly synthesized brain DNA. Physiol Behav 43:339-350.

Bliss TVP, Collingridge GL (1993) A synaptic model of memory: longterm potentiation in the hippocampus. Nature 361:31-39.

Bloch V, Laroche S (1981) Conditioning of hippocampal cells: its acceleration and long-term facilitation by post-trial reticular stimulation. Behav Brain Res 3:23-42.

Bloch V, Laroche S (1984) Facts and hypotheses related to the search for the engram. In: Neurobiology of learning and memory (Lynch G, McGaugh JL, Weinberger NM, eds), pp 249-260. New York: Guilford.

Bloch V, Laroche S (1985) Enhancement of long-term potentiation in the rat dentate gyrus by post-trial stimulation of the reticular formation. J Physiol (Lond) 360:215-231.

Bloch V, Hennevin E, Leconte P (1977) Interaction between post-trial reticular stimulation and subsequent paradoxical sleep in memory consolidation processes. In: Neurobiology of sleep and memory (DruckerColin RR, McGaugh JL, eds), pp 255-272. New York: Academic.

Bramham CR, Srebro B (1989) Synaptic plasticity in the hippocampus is modulated by behavioral state. Brain Res 493:74-86.

Bramham CR, Maho C, Laroche S (1994) Suppression of long-term potentiation induction during alert wakefulness but not during "enhanced" REM sleep after avoidance learning. Neuroscience 59:501-509.

Brooks DC, Bizzi E (1963) Brain stem electrical activity during deep sleep. Arch Ital Biol 101:648-665.

Buzsaki G (1989) Two-stage model of memory trace formation: a role of "noisy" brain states. Neuroscience 31:551-570.

Buzsaki G (1996) The hippocampo-neocortical dialogue. Cereb Cortex 6:81-92.

Datta S (1995) Neuronal activity in the peribrachial area: relationship to behavioral state control. Neurosci Biobehav Rev 19:67-84.

Datta S (1997) Cellular basis of pontine ponto-geniculo-occipital wave generation and modulation. Cell Mol Neurobiol 17:341-365.

Datta S (1999) PGO wave generation: mechanisms and functional significance. In: Rapid eye movement sleep (Mallick BN, Inoue S, eds), pp 91-106. New Delhi, India: Narosa.

Datta S, Hobson AJ (1994) Neuronal activity in the caudolateral peribrachial pons: relationship to PGO waves and rapid eye movements. J Neurophysiol 71:95-109.

Datta S, Hobson AJ (1995) Suppression of ponto-geniculo-occipital waves by neurotoxic lesions of pontine caudo-lateral peribrachial cells. Neuroscience 67:703-712.

Datta S, Calvo JM, Quatrochi JJ, Hobson JA (1992) Cholinergic microstimulation of the peribrachial nucleus in the cat. I. Immediate and prolonged increases in ponto-geniculo-occipital waves. Arch Ital Biol 130:263-284.

Datta S, Siwek DF, Patterson EH, Cipolloni PB (1998) Localization of pontine PGO wave generation sites and their anatomical projections in the rat. Synapse 30:409-423.

Datta S, Patterson EH, Siwek DF (1999) Brainstem afferents of the cholinoceptive pontine wave generation sites in the rat. Sleep Res Online 2:79-82.

Destrade C, Cardo B (1974) Effects of post-trial hippocampal stimulation on time-dependent improvement of performance in mice. Brain Res 78:447-454.

Destrade C, Soumireu-Mourat B, Cardo B (1973) Effects of posttrial hippocampal stimulation on acquisition of operant behavior in the mouse. Behav Biol 8:713-724.

Devietti TL, Conger GL, Kirkpatrick BR (1977) Comparison of the enhancement gradients of retention obtained with stimulation of the mesencephalic reticular formation after training or memory reactivation. Physiol Behav 19:549-554. 
DeWeer B (1976) Selective facilitative effect of post-trial reticular stimulation in discriminative learning in the rat. Behav Processes 1:243-257.

Dujardin K, Guerrien A, Leconte P (1990) Sleep, brain activation and cognition. Physiol Behav 47:1271-1278.

Erickson CK, Patel JB (1969) facilitation of avoidance learning by posttrial hippocampal electrical stimulation. J Comp Physiol Psychol 68:400-406.

Fishbein W (1971) Disruptive effects of rapid eye movement sleep deprivation on long term-memory. Physiol Behav 6:279-282.

Fishbein W, Gutwein BM (1977) Paradoxical sleep and memory storage processes. Behav Biol 19:425-464.

Fishbein W, Kastaniotis C, Chattman D (1974) Paradoxical sleep: prolonged augmentation following learning. Brain Res 79:61-75.

Gallagher M, McMahan RW, Schoenbaum G (1999) Orbitofrontal cortex and representation of incentive value in associative learning. J Neurosci 19:6610-6614.

Hars B, Hennevin E (1987) Impairment of learning by cueing during post-learning slow-wave sleep in rats. Neurosci Lett 79:290-294.

Hars B, Hennevin E, Pasques P (1985) Improvement of learning by cueing during post-learning paradoxical sleep. Behav Brain Res 18:241-250.

Hatfield T, Han J-S, Conley M, Gallagher M, Holland P (1996) Neurotoxic lesions of basolateral, but not central, amygdala interfere with pavlovian second-order conditioning and reinforcer devaluation effects. J Neurosci $16: 5256-5265$.

Hennevin E, Hars B (1985) Post-learning paradoxical sleep: a critical period when new memory is reactivated. In: Advances in behaviora biology, Vol 28, Brain plasticity, learning and memory (Will BE, Schmitt P, Dalrymple-Alford J-C, eds), pp 193-203. New York: Plenum.

Hennevin E, Hars B, Bloch V (1989) Improvement of learning by mesencephalic reticular stimulation during postlearning paradoxical sleep. Behav Neural Biol 51:291-306.

Hennevin E, Hars B, Maho C, Bloch V (1995) Processing of learned information in paradoxical sleep: relevance for memory. Behav Brain Res 69:125-135.

Izquierdo I, Fin C, Schmitz PK, DaSilva RC, Jerusalinsky D, Quillfeldt JA, Ferreira MBG, Medina JH, Bazan NG (1995) Memory enhancement by intrahippocampal, intraamygdala, or intraentorhinal infusion of platelet-activating factor measured in an inhibitory avoidance task. Proc Natl Acad Sci USA 92:5047-5051.

Jones-Leonard B, McNaughton BL, Barnes CA (1987) Suppression of hippocampal synaptic plasticity during slow-wave sleep. Brain Res 425:174-177.

Joy RM, Prinz PN (1969) The effect of sleep-altering environments upon the acquisition and retention of a conditioned avoidance response in the rat. Physiol Behav 4:809-814.

Karni A, Tanne D, Rubenstein BS, Askenasy JJ, Sagi D (1994) Dependence on REM sleep of overnight improvement of a perceptual task. Science 265:679-682.

Kudrimoti HS, Barnes CA, McNaughton BL (1999) Reactivation of hippocampal cell assemblies: effects of behavioral state, experience, and EEG dynamics. J Neurosci 19:4090-4101.

Landfield PW, Tusa RJ, McGaugh JL (1973) Effects of posttrial hippocampal stimulation memory storage and EEG activity. Behav Biol 8:485-505

Laurent JP, Ayalaguerrero F (1975) Reversible suppression of pontogeniculo-occipital waves by localized cooling during paradoxical sleep in cats. Exp Neurol 49:356-369.

Leconte P, Hennevin E, Bloch V (1974) Duration of paradoxical sleep necessary for the acquisition of conditioned avoidance in the rat. Physiol Behav 13:675-681.

LeDoux JE (1992) Emotion and the amygdala. In: The amygdala: neurobiological aspects of emotion, memory, and mental dysfunction (Aggleton J, ed), pp 339-351. New York: Wiley.

Lucero M (1970) Lengthening of REM sleep duration consecutive to learning in a rat. Bran Res 20:319-322.

Maho C, Bloch V (1992) Responses of hippocampal cells can be conditioned during paradoxical sleep. Brain Res 581:115-122.

Marks GA, Farber J, Rubinstein M, Roffwarg HP (1980) Demonstration of ponto-geniculo-occipital waves in the albino rat. Exp Neurol 69:648-655

McGrath MJ, Cohen DB (1978) REM sleep facilitation of adaptive waking behavior: a review of the literature. Psychol Bull 85:24-57.

Nelson JP, McCarley RW, Hobson JA (1983) REM sleep burst neurons, PGO waves, and eye movement information. J Neurophysiol 50:784-797.

Pavlides C, Winson J (1989) Influences of hippocampal place cell firing in the awake state on the activity of these cells during subsequent sleep episodes. J Neurosci 9:2907-2918.
Paxinos G, Watson C (1986) The rat brain in stereotaxic coordinates. New York: Academic.

Pearlman C (1973) REM sleep deprivation impairs latent extinction in rats. Physiol Behav 11:233-237.

Pearlman C (1979) REM sleep and information processing: evidence from animal studies. Neurosci Biobehav Rev 3:57-68.

Pearlman C, Becker M (1973) Brief posttrial REM sleep deprivation impairs discrimination learning in rats. Physiol Psychol 1:373-376.

Poremba A, Gabriel M (1997) Amygdalar lesions block discriminative avoidance learning and cingulothalamic training-induced neuronal plasticity in rabbits. J Neurosci 17:5237-5244.

Portell-Cortes I, Marti-Nicolovius M, Segura-Torres P, Morgado-Bernal I (1989) Correlations between paradoxical sleep and shuttlebox conditioning in rats. Behav Neurosci 103:984-990.

Qin Y, McNaughton BL, Skaggs WE, Barnes CA (1997) Memory reprocessing in cortico-cortical and hippocampo-cortical neuronal ensembles. Philos Trans R Soc Lond [Biol] 352:1525-1533.

Ramirez OA, Carrer HF (1989) Correlation between threshold to induce long-term potentiation in the hippocampus and performance in a shuttle box avoidance response in rats. Neurosci Lett 104:152-156.

Rempel-Clower NL, Zola SM, Squire LR, Amaral DG (1996) Three cases of enduring memory impairment after bilateral damage limited to the hippocampal formation. J Neurosci 16:5233-5255.

Sakai K, Petitjean F, Jouvet M (1976) Effects of pontomesencephalic lesions and electrical stimulation upon PGO waves and EMPs in unanesthetized cats. Electroencephalogr Clin Neurophysiol 41:49-63.

Sanford LD, Tejani-Butt SM, Ross RJ, Morrison AR (1995) Amygdaloid control of alerting and behavioral arousal in rats: involvement of serotonergic mechanisms. Arch Ital Biol 134:81-99.

Sara SJ, Deweer B, Hars B (1980) Reticular stimulation facilitates retrieval of a "forgotten" maze habit. Neurosci Lett 18:211-217.

Silva AJ, Stevens CF, Tonegawa S, Wang Y (1992) Deficient hippocampal long-term potentiation in alpha-calcium-calmodulin kinase II mutant mice. Science 257:201-206.

Skaggs WE, McNaughton BL (1996) Replay of neuronal firing sequences in rat hippocampus during sleep following spatial experience. Science 271:1870-1873.

Smith C (1985) Sleep states and learning: a review of the animal literature. Neurosci Biobehav Rev 9:157-168.

Smith C, Butler S (1982) Paradoxical sleep at selective times following training is necessary for learning. Physiol Behav 29:469-473.

Smith C, Kelly G (1988) Paradoxical sleep deprivation applied two days after end of training retards learning. Physiol Behav 43:213-216.

Smith C, Rose GM (1997) Posttrainning paradoxical sleep in rats is increased after spatial learning in the Morris water maze. Behav Neurosci 111:1197-1204.

Smith C, Wong PTP (1991) Paradoxical sleep increases predict successful learning in a complex operant task. Behav Neurosci 105:282-288.

Smith C, Young J (1980) Reversal of paradoxical sleep deprivation by amygdaloid stimulation during learning. Physiol Behav 24:1035-1039.

Smith C, Young J, Young W (1980) Prolonged increases in paradoxical sleep during and after avoidance task acquisition. Sleep 3:67-81.

Smith C, Conway JM, Rose GM (1998) Brief paradoxical sleep deprivation impairs reference, but not working, memory in the radial arm maze task. Neurobiol Learn Mem 69:211-217.

Squire LR, Amaral DG, Press GA (1990) Magnetic resonance imaging of the hippocampal formation and mammillary nuclei distinguish medial temporal lobe diencephalic amnesia. J Neurosci 10:3106-3117.

Stein DG, Chorover S (1968) Effects of post-trial electrical stimulation of hippocampus and caudate nucleus on maze learning in the rat. Physiol Behav 3:787-791.

Stickgold R (1998) Sleep: off-line memory reprocessing. Trends Cogn Sci $2: 484-492$.

Vazdarjanova A, McGaugh JL (1999) Basolateral amygdala is involved in modulating consolidation of memory for classical fear conditioning. J Neurosci 19:6615-6622.

Vertes RP (1984) Brainstem control of the events of REM sleep. Prog Neurobiol 22:241-288.

Wilson MA, McNaughton BL (1994) Reactivation of hippocampal ensemble memories during sleep. Science 265:676-679.

Wolfowitz BE, Holdstock TL (1971) Paradoxical sleep deprivation and memory in rats. Commun Behav Biol 6:281-284.

Young B, Otto T, Fox GD, Eichenbaum H (1997) Memory representation within the parahippocampal region. J Neurosci 17:5183-5195. 